\title{
Protein microarray velcro
}

A designed polypeptide scaffold contains a surface immobilization domain and a target capture domain for flexible protein arraying.

'Microarray' might just be the hottest buzzword in biology today. Small molecules, oligonucleotides and proteins are being immobilized onto surfaces for various high throughput screening studies. There are unique chemical challenges in the immobilization of each type of molecule onto a surface, yet as the technology advances, thankfully there are more and more available solutions.

Protein immobilization on a microarray surface is especially tricky because of the inherent sensitivity of proteins to perturbations. "The display of proteins in microarray format is a problem for which there is no general solution yet," remarks Caltech professor David Tirrell. Initial efforts to construct protein arrays exploited the inherent reactivity of lysine or cysteine residues to covalently capture pro- teins with a reactive group on the surface, but this can cause active sites to become unavailable, or it can even prompt denaturation of the protein. Many researchers since have borrowed traditional affinity chromatography methods to display proteins on surfaces, such as fusing the protein of interest with a hexahistidine tag for capture by immobilized metal or biotinylating the protein for capture by avidin/streptavidin. The challenge still remains, however, to construct tags that are extremely stable under a variety of conditions, while maintaining high affinity for the surface chemistry. Toward that goal, Tirrell and colleagues recently introduced a new flexible polypeptide scaffold consisting of a surface immobilization domain and a protein capture domain.

The immobilization domain is composed of a highly hydrophobic elastin mimetic peptide that strongly adheres to hydrophobic surfaces. Furthermore, incorporation of an unnatural photoreactive

\section{SWITCHING CHANNELS}
A light-controlled protein channel may represent the prototype for a new generation of nanotechnology tools.
Conceptually, building a
nanomachine isn't entirely different from building any other device- the engineer needs to first design the necessary parts and then determine how to assemble them to achieve the desired effect. The tricky part, of course, comes from working at the nanoscale, where efficiency of design is a must. Fortunately, evolution has successfully tackled countless engineering challenges, and inspiration can often be found somewhere in the natural protein world.
Researchers at the University of Groningen and the BiOMaDe Technology Foundation in the Netherlands recently demonstrated the power of this
approach; they reengineering $\mathrm{MscL}-\mathrm{a}$ homopentameric mechanosensitive channel expressed in Escherichia coli-so that it can be reversibly opened or closed in response to light. "In the natural system, it is a safety valve," explains author Ben Feringa. "It prevents the cell from bursting - so if there's too much pressure in the cell, suddenly the pore opens three nanometers, and a lot of material gets out of the cell. So it's a very nice channel that can be opened naturally, and the idea was to open or close it on command."
MscL is normally tightly sealed by hydrophobic interactions; however, by introducing sufficient charge, the pore can be compelled to open- and remain so until the charge is removed. With this in mind, Feringa and his colleagues engineered a reversible 


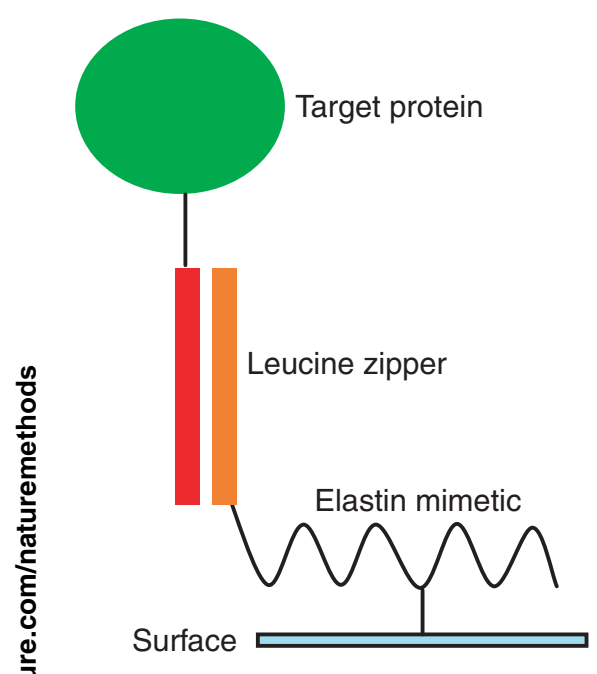

phenylalanine derivative allows the scaffold to be covalently linked to an appropriately derivatized surface upon UV irradiation. The protein capture domain consists of one helix of a leucine zipper coiled coil. The complementary helix is expressed as a fusion with the target protein of interest. Owing to the extremely tight and specific noncovalent association of the leucine zipper coiled coil, the protein of interest
Figure 1 | A new polypeptide scaffold for flexible protein arraying consists of an elastin mimetic domain and a leucine zipper helix. The elastin mimetic domain is covalently bound to the surface by a photoreactive amino acid. The target protein is fused to the complementary leucine zipper helix; strong noncovalent association of the helices leads to dimerization, effectively immobilizing the target protein.

can be efficiently captured by the immobilized scaffold (Fig. 1), even from a crude cell lysate.

The engineered polypeptide scaffold allows much greater flexibility in the immobilization of proteins on a microarray for functional studies. "Because proteins have such variable chemistries, it is difficult to array diverse sets of proteins," explains Tirrell. "We are able to link proteins to a surface, but we are also able to provide some diversity in the nature of the linkage by using protein design."

\section{Allison Doerr}

\section{RESEARCH PAPERS}

Zhang, K. et al. Artificial polypeptide scaffold for protein immobilization. J. Am. Chem. Soc. 127, 10136-10137 (2005). photoswitch, which undergoes conversion to a charged state after exposure to UV light, but is neutralized by visible wavelengths. This switch was attached at a key point in the MscL monomer, and the engineered protein was inserted into synthetic membranes. Patch-clamp experiments confirmed that after a brief lag, UV light induced opening of the channels, and they remained open until being rapidly resealed by visible light. A second round of experiments demonstrated that, minor leakage aside, light could effectively regulate the release of the fluorescent dye calcein from liposomes containing the engineered MscL.

These findings are preliminary, but hold promise for future refinement of this approach. One application for which the authors envision using these engineered pores is as a tool for regulating drug delivery, and the BiOMaDe team is now exploring this possibility. Feringa sees an even bigger role for these little machines, however, and believes they could prove useful as elements of sophisticated nanodevices. "We have hardly any idea in the field of nanotechnology how to integrate components, how to assemble them and keep them working properly...," he says. "With the principle proven, there's a nice challenge to see if we can integrate such nanovalves with, say, nanofluidics-some channels where this would actually function as a valve."

\section{Michael Eisenstein}

\section{RESEARCH PAPERS}

Koçer, A. et al. A light-activated nanovalve derived from a channel protein. Science 309, 755-758 (2005). 